\title{
Level 5: Gaming als Bestandsthema?
}

\section{Vertriebsplattformen und ihre Bedeutung für Bibliotheken}

Eine klassische Bestandsarbeit wird im Bereich Gaming für Bibliotheken immer schwieriger werden. Im nachfolgenden Abschnitt gehe ich explizit auf das Thema Bestandsarbeit ein. In diesem Abschnitt geht es nun um die digitalen Vertriebsplattformen für Computerspiele. In den nächsten zehn Jahren wird der physische Datenträger verschwinden oder höchstens noch ein Nischenthema sein. Dies betrifft nicht alleine die Games. Auch die Bücher und viele weitere analoge Medien werden einen tiefgreifenden Wandel erleben. Für Bibliotheken bedeutet dies, dass sich ihre Arbeit nachhaltig verändern wird. Der Bestand einer Bibliothek wird an Bedeutung verlieren. Im Bereich Gaming ist dieser Wandel schon länger im Gange und er hat bereits Formen angenommen, die mit Sicherheit auch in den anderen für Bibliotheken relevanten Bereichen Realität werden.

Der digitale Vertrieb von Computergames wird über verschiedene Plattformen abgewickelt. Im Folgenden werde ich die relevanten Plattformen Origin, Steam, Playstation Network, XBOX Live und Humble Bumble beschreiben. Allen Plattformen gemein ist die Möglichkeit, Spiele direkt auf der Plattform zu kaufen und dann herunterzuladen. Wenn ich ein Spiel gekauft habe, erscheint es in meiner „Bibliothek“ und von dort kann ich es runterladen. In allen Fällen ist ein Account bei der Plattform nötig. In immer mehr Fällen ist dieser Account auch notwendig, wenn man ein Spiel auf einem physischen Datenträger gekauft hat. Einige Plattformen dienen nämlich auch als Aktivierungssystem. Der Spieler kann das Spiel nur dann spielen, wenn er es auf der jeweiligen Plattform aktiviert. Das Spiel ist also fest verbunden mit dem jeweiligen Benutzerkonto. Dies macht auch einen eventuellen Weiterverkauf schwierig bis unmöglich. Hierfür wird wie auch bei vielen eBooks ein sogenantes Digitales Rechtemanagement (DRM) genutzt.

Die Plattformen sind manchmal auch Communities. Man kann als Spieler sehen, wer von den vernetzten Kontakten welches Spiel spielt. Man kann ein eigenes Profil anlegen und sich mit anderen Spielern austauschen.

Die in manchen Fällen nicht vorhandene Möglichkeit, die Spiele weiterverkaufen zu können, und immer wieder aufkommende Fragen bezüglich des Datenschutzes sind immer wieder Gegenstand von kritischen Diskussionen. Andererseits ergeben sich für die Spieler auch Vorteile. So fungiert die jeweilige Plattform auch als Sicherungskopie. Wenn z.B. die Festplatte kaputt geht, kann man die Spiele erneut herunterladen. Zudem werden auch die jeweiligen Spielstände gespeichert, was bedeutet, dass man das Spiel nicht von vorne beginnen muss. Darüber hinaus werden über manche Plattformen auch Spiele-Updates zur Verfügung gestellt.

Kommen wir nun zu den einzelnen Plattformen:

\section{Origin}

Origin ist die Gaming-Vertriebsplattform von Electronic Arts (EA). Man kann auf dieser Plattform Spiele kaufen und downloaden. Die Plattform ist ebenso ein OnlineSpeicher für die gekauften Spieler. Man kann ein eigenes Profil mit Bild etc. erstellen und sich mit anderen Spielern „befreunden“. Somit verfügt Origin über rudimentäre Community-Funktionen. Origin ist aber ebenso die Verwaltungsplattform für das DRM der einzelnen Spiele. Das bedeutet, dass viele Spiele von EA nur dann gespielt werden können wenn sie auf Origin aktiviert werden. Nachdem man ein Spiel online gekauft hat, kann man es über einen Client downloaden. 


\section{Steam}

Steam ist eine weitere und sehr erfolgreiche Plattform für den digitalen Vertrieb bzw. das digitale Management von Computerspielen und weiterer Software. Hinter Steam steht das US-amerikanische Unternehmen Valve. Wie auch bei Origin kann man auch hier Spiele kaufen und herunterladen. Die Plattform ist ebenso für die Aktivierung sehr vieler Spiele notwendig. Jeder Nutzer hat einen eigenen Account. Die gekauften Spiele erscheinen in der eigenen Bibliothek und können von dort heruntergeladen werden. Über Steam werden die einzelnen Spiele auch aktualisiert. Besonders interessant ist die gut ausgebaute Community, die nicht nur die Kommunikation zwischen den Spielern ermöglicht. Unter dem Namen „Steam Greenlight“ können „kleine“ Entwickler ihre Spieler der Community vorstellen und ein Feedback bekommen. Zudem ermöglicht die Community das Teilen von Spielerweiterungen, die von Entwicklern oder aber anderen Gamern entwickelt wurden.

\section{Humble Bundle}

Es gibt viele interessante Ansätze für neuartige Vertriebskonzepte. Und doch ist Humble Bundle etwas Besonderes. Wie bei den bereits beschriebenen Plattformen auch, kann man auf dieser Plattform Spiele kaufen. Das Besondere ist die Tatsache, dass man selber entscheidet, wie viel man für die Spiele bezahlen möchte. In vielen Fällen kann man aber nicht nur den Anbietern der Spiele Geld überweisen. Sehr oft gibt es zudem die Möglichkeit, für gemeinnützige Organisation zu spenden. Hierfür definiert man zuerst den Betrag, den man insgesamt zahlen möchte, und kann dann die Aufteilung der Gelder auf den Anbieter des Spiels, die Plattform und die gemeinnützigen Organisationen festlegen. An dieser Plattform ist weniger die technische Plattform als vielmehr das damit verbundene Bezahlmodell interessant. Vor allem für Independent-Entwickler ist diese Plattform spannend. Zumindest dann, wenn ihr Spiel gut ist und sie eine Fan-Gemeinde aufbauen können.

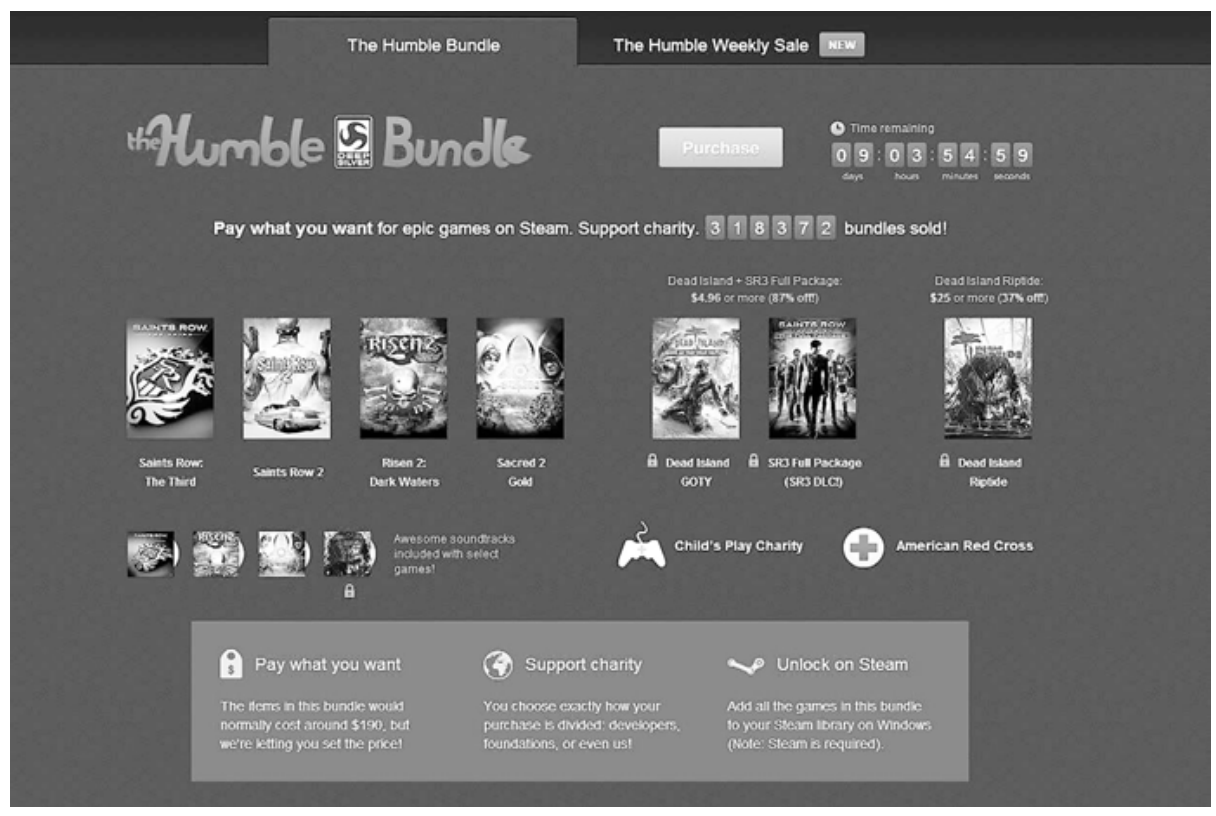

Abb. 24 Screenshot der Plattform Humble Bundle 


\section{Nintendo eShop, Playstation Store, XBOX Live}

Natürlich haben auch die drei Konsolenanbieter eigene Verkaufsplattformen aufgebaut. Sie sind ein elementarer Bestandteil der jeweiligen Konsolensoftware. Über seinen Account kann der Spieler Spiele, Add-Ons, Trailer und viele weitere Angebote kaufen bzw. herunterladen. Sony bietet zudem ein Abonnement an, bei dem die Spieler gegen eine Jahresgebühr eine Vielzahl an aktuellen Spielen kostenlos herunterladen und spielen können. Solange das Abonnement läuft, besteht auch der Zugriff auf die Spiele.

\section{App-Stores}

Natürlich wird auch auf Smartphones und Tablets gespielt. Die Spiele werden dann über die jeweiligen App-Stores vertrieben. Diese Stores haben keine Communityfunktion und dienen einzig dem Vertrieb von Apps inkl. der Spiele. Es gibt sowohl kostenlose als auch kostenpflichtige Spiele. Das Modell ist dabei sehr einfach. Jeder Eigentümer eines Smartphones oder eines Tablets hat ein Konto im App-Store. Die Spiele werden über dieses Konto bezahlt. Ein Weiterverkauf ist nicht möglich. Allerdings können die Spiele auch auf andere Devices runtergeladen werden, wenn diese Devices ebenfalls an das Konto angebunden werden.

Wenn wir uns die beschriebenen Plattformen genauer anschauen, wird schnell klar, dass es für Bibliotheken keine Möglichkeit gibt, ein eigenes Angebot zu entwickeln. Es wird also keine „Gaming-Onleihe“ geben. Die genannten Plattformen haben aber auch Einfluss auf die Bestandsarbeit mit physischen Datenträgern, denn wenn Spiele an einen Account gebunden sind, lassen sie sich schlecht oder gar nicht verleihen. Für die Anbieter der Spiele macht es in der Zukunft keinen Sinn mehr, Spiele auf physischen Datenträgern zu verkaufen. Die Kosten für die Produktion und den physischen Vertrieb sind einfach zu hoch. Zudem lassen sich die Kunden viel besser an die Spiele binden, wenn Plattformen wie Steam oder Origin für die Aktivierung notwendig sind, und wenn zudem weitere Inhalte für die Spiele nur über Onlineplattformen heruntergeladen werden können. Der Verkauf von gebrauchten Spielen wird erschwert. Für viele kleine Entwickler ist der physische Vertrieb gar keine Option. Sie benötigen den digitalen Vertrieb, um überhaupt ihre Spiele verkaufen zu können.

Es gibt noch weitere Plattformen, vor allem im Bereich der Independent Games. Jedoch haben diese Plattformen insgesamt wenig Relevanz. Aus diesem Grund habe ich sie nicht in die Sammlung aufgenommen. Auf meinem Blog bzw. dem Wiki zum Buch werden sie aber zu finden sein.

\section{Bestandsfragen}

Viele Bibliotheksmitarbeiter denken beim Thema Gaming zuerst an den Bestand. Dies ist natürlich nachvollziehbar. Eine Bibliothek ohne Bestand ist für sehr viele Menschen undenkbar. Und die Auswahl, Beschaffung, Vermittlung und das Zugänglichmachen von unterschiedlichen Medien und Inhalten sind mit Sicherheit auch in der Zukunft wichtige bibliothekarische Aufgaben. Der Bestand einer Bibliothek ist zudem noch immer ein entscheidendes Kriterium für die Anzahl und Besoldung von Stellen und die Bereitschaft in die Infrastruktur einer Bibliothek zu investieren. Für viele Menschen ist eine Bibliothek noch immer ein Ort, an dem man primär Bücher und in Teilen auch andere Medien wie z. B. Filme und Musik ausleihen kann. Auch viele Trä- 
ger sind dieser Meinung und es sind sehr oft genau diese Träger, die nicht verstehen können, warum sich eine Bibliothek aktiv mit dem Thema Gaming und ähnlichen Themen wie z. B. Social Media beschäftigen sollte.

Gleichwohl sind Bibliotheken keine reinen „Verleih-Stationen“ mehr. In den meisten Fällen bieten sie eine Vielzahl an weitergehenden Services an. Unabhängig von der Ausrichtung als wissenschaftliche oder öffentliche Bibliothek wird versucht, den Nutzern/Kunden in vielen weiteren Lebenssituationen zu helfen: Von der Leseförderung und Autorenlesungen bis hin zur Vermittlung von Recherchekompetenz sind Bibliotheken in vielen Feldern aktiv. In immer mehr Fällen wird die Bibliothek zum Lern-, Kultur- und/oder Sozialort. Gleichwohl ist der Bestand einer Bibliothek noch immer das zentrale Thema. Das bedeutet, dass es für Bibliotheken noch immer sehr schwer ist, ein Thema zu bearbeiten, ohne einen Bezug zum eigenen Bestand herstellen zu können. So entstehen Aussagen wie: „Wir machen was mit Computerspielen, um andere Zielgruppen in die Bibliothek zu locken, damit wir sie dann mit den Büchern in Kontakt bringen“. Die Idee ist nachvollziehbar, aber sie wird nicht funktionieren. Wenn Sie als Bibliothek das Thema Gaming in den Bestand aufnehmen oder aber den vorhandenen Bestand ausbauen möchten, dann müssen Sie Gaming und Games als gleichwertig zu Büchern, Filmen und Musik akzeptieren. Games sind kein Thema, um Menschen zum Lesen von Büchern zu bringen.

So wie sich unsere Gesellschaft sowohl analog als auch digital stetig weiter- entwickelt, müssen und sollten dies auch die Bibliotheken tun. Und gerade die digitale Welt steht für eine Vielzahl an Chancen und Herausforderungen für Bibliotheken sowohl im wissenschaftlichen als auch im öffentlichen Bereich. Und denken Sie immer an die Aussagen, welche ich zu Beginn des Buches gemacht habe: Gaming ist Lesen, Gaming ist Lernen. Ohne Games bzw. Gaming ist eine digitale Strategie einer Bibliothek, und dies gilt sowohl für wissenschaftliche als auch für öffentliche Bibliotheken, unvollständig und wird früher oder später an ihre Grenzen stoßen.

Gaming sollte m.E. ein elementarer Bestandteil der bibliothekarischen Arbeit sein und der Bestand ist ein wesentlicher Teil einer Bibliothek. Was spricht also dagegen, auch im Bereich Gaming einen eigenen Bestand aufzubauen bzw. zu erweitern? Die Antwort ist: Nichts, aber...

Grundsätzlich ist es natürlich eine gute Idee, einen eigenen Gamingbestand zu haben. Viele Bibliotheken sind damit sehr erfolgreich. Und für einige Gamer ist ein gut sortierter Bibliotheksbestand ebenfalls von Vorteil. Der Teufel steckt wie so oft im Detail. Der Aufbau eines guten und nachhaltig erfolgreichen Gaming-Bestandes ist alles andere als einfach. Im Folgenden möchte ich auf die Gründe für diese Situation und die m. E. vorhandenen Optionen für Bibliotheken eingehen.

Ein Gamingbestand ist sicherlich eine tolle Sache. Aber nicht nur bei diesem Thema stößt die klassische Bibliotheksarbeit zunehmend an ihre Grenzen. Die kontinuierlich fortschreitende Digitalisierung unserer Gesellschaft ist vor allem im Bereich der Digitalisierung der Medien zu beobachten. In den letzten Jahren hat sich sowohl das Angebot als auch das Mediennutzungsverhalten vieler Menschen nachhaltig verändert. Physische Träger haben eine starke Konkurrenz bekommen. Filme, Musik, Bücher etc. sie alle werden mehr und mehr durch elektronische bzw. digitale Formen abgelöst. Wahrscheinlich wird es auch in Zukunft noch gedruckte Bücher, Schallplatten, DVDs etc. geben aber ihre Bedeutung wird massiv abgenommen haben.

Wie die Zukunft der Medien im Detail aussehen wird, kann heute niemand voraussagen. Dass wir aber in den nächsten Jahren die fortschreitende Digitalisierung unserer Gesellschaft und damit verbunden unserer Medien erleben werden, wird von niemandem mehr ernsthaft bestritten. Vor allem im Bereich Gaming ist dies sehr gut zu beobachten. Die Zukunft der Games liegt nicht im physischen Datenträger. 
Die Vertriebsmodelle der Gaming-Industrie verändern sich rasant. Da ist zum einen das Download-Geschäft. Immer mehr Spiele werden direkt auf den jeweiligen PC, die jeweilige Konsole oder aber auf ein Smartphone oder einen Tablet-PC runtergeladen. Alle genannten Geräte verfügen in der Regel über ausreichende Speicherkapazitäten, um eine Vielzahl an Spielen spielen zu können. Auch beim PC können wir ähnliche Tendenzen beobachten. Bei Spielen auf dem iPad und Smartphones findet der Vertrieb bereits ausschließlich über Downloads aus den jeweiligen Stores statt. Diese Downloads sind in der Regel mit Kosten verbunden und manch einer mag nun überlegen, ob sich hier nicht eine Chance für Bibliotheken für eine neue Bestandsarbeit auftut. Aber dem ist nicht so. Im Gegensatz zu den eBooks, die in Teilen auch von Bibliotheken verliehen werden können, ist diese Option im Bereich Gaming nicht zu erwarten. Manche Spiele werden schon jetzt nur noch via Download angeboten, d. h. diese Spiele können definitiv nicht mehr auf einem physischen Datenträger erworben werden. Damit können Sie auch nicht Teil des physischen Bestands einer Bibliothek werden. Die Downloads werden wiederum zumeist über spezielle OnlineVertriebs-Plattformen wie z. B. Steam oder Origin abgewickelt. Bei Smartphones und Tablet-PCs sind die Spiele Apps, welche im jeweiligen App-Store zu bekommen sind. In allen genannten Fällen wird es mit an Sicherheit grenzender Wahrscheinlichkeit keine Angebote von Bibliotheken geben oder anders ausgedrückt: es gibt keine Gaming-Onleihe

Neben dem Verschwinden des physischen Datenträgers und dem digitalen Vertrieb von Games über Onlineplattformen entstanden und entstehen parallel noch weitere und zugleich völlig neue Geschäftsmodelle auf Seiten der Anbieter, die mit der klassischen Bestandsarbeit einer Bibliothek erst recht nicht mehr kompatibel sind. So werden immer mehr Computerspiele online gespielt. In vielen Fällen werden diese Spiele im Browser gespielt, das heißt der Internet-Browser wird zum Zugang zum jeweiligen Spiel. Hier gibt es überhaupt keine Verleihmöglichkeit mehr, da man das Spiel nicht kauft oder leiht, sondern nur Zugang zur Spielewelt bekommt. In anderen Fällen lädt man ein kleines Programm, einen sogenannten Client, herunter, der dann das Tor zur Spielewelt ist. In beiden Fällen ist eine Bestandsarbeit nahezu unmöglich oder wäre, wenn man es unbedingt umsetzen wollte, mit extrem hohen Kosten verbunden. Selbst das sehr oft vorhandene Alleinstellungsmerkmal, dass Bibliotheken den kostenlosen Zugang zu Inhalten ermöglichen, welche sonst nur gegen Geld erworben werden können, lässt ich auf den Gaming-Bereich immer seltener anwenden. Denn ein verhältnismäßig junges Geschäftsmodell ist Freemium. Dabei ist das Spielen der Spiele komplett kostenlos. Erst wenn ich beispielsweise im Spiel besondere Vorteile haben möchte oder aber meine Spielfigur gesondert ausstatten möchte etc., erst dann fallen Kosten an. Des Weiteren gibt es viele sogenannte SocialGames, die als Teil von Facebook oder anderen Plattformen ebenfalls den Zugang kostenlos ermöglichen und dann entweder durch In-Game-Werbung oder aber wie eben schon beschrieben, durch spezielle kostenpflichtige Features Geld verdienen.

Auch diesen Bereich der Gaming-Welt können wir nicht in klassischer Form in einem Bestand einer Bibliothek erfassen. Schließlich gibt es noch ein weiteres Problem: die Dauer der Spiele. Es gibt einige Spiele, die man linear also von ihrem Beginn bis zum Schluss spielt und die dann abgeschlossen sind. Man kann das Spiel nach dem Spielen ohne weiteres zurückgeben, es ist ja zu Ende gespielt worden. Sehr viele Spiele sind aber nicht endlich oder es dauert sehr lange, bis man das Spiel wirklich beendet hat. Dies gilt auch und vor allem für alle Spiele, die im Multiplayer-Modus gespielt werden. Gerade diese Spiele weisen sehr oft nur eine sehr schwache Solo-Kampagne auf. Wenn man ein Spiel aber online gegen andere spielen will, dann muss es letztlich dauerhaft beim Spieler vorhanden sein, was gegen das Verleihmodell spricht. Es gibt 
aber auch Spiele, die schlichtweg zu komplex und damit zu groß sind, um sie innerhalb von ein bis zwei Wochen durchzuspielen.

Bedeutet dies, dass man als Bibliothek im Bereich Gaming auf die Bestandsarbeit verzichten sollte? Die Antwort ist ein klares Nein! Aber es ist wichtig, dass Sie sich für den Bestandsaufbau eine neue Strategie überlegen. Natürlich kenne ich nicht alle individuellen Bestandsstrategien aller Bibliotheken im Bereich Gaming. Jedoch habe ich in meinen vielen Workshops und Gesprächen drei Trends ausmachen können:

1. Bibliotheken beschäftigen sich in der Regel zu wenig mit dem Thema Gaming und sind damit kaum in der Lage, eine nachhaltige und umfassende Bestandsarbeit zu gewährleisten. In diesen Fällen findet zumeist eine Fokussierung auf neue und vermeintlich erfolgreiche Spiele statt. Es erinnert dann ein bisschen an die Bestsellerlisten im Bereich Buch.

2. Da die in Punkt 1 genannten Schwierigkeiten auftreten, wird manchmal versucht auf Standing Orders zurückzugreifen.

3. Die Zielgruppendefinition ist falsch. Das Thema Gaming wird sehr oft für die Zielgruppe von Kindern, Jugendlichen und jungen Erwachsenen umgesetzt. Wie ich aber bereits beschrieben habe, ist das Thema für weitaus mehr Altersstufen interessant. Hier wird sehr oft großes Potential verschenkt.

Ich rate dringend davon ab, das Thema Gaming über Standing Orders abzuwickeln. An sich bin ich ein großer Fan dieses Modells, denn Bibliotheken können auf diesem Weg Ressourcen einsparen, die man für andere Aktivitäten z. B. Leseförderung, Social Media etc. nutzen kann. Beim Thema Gaming ist dies aber problematisch. Games sind etwas komplett anderes als Filme oder gar Bücher. Sie sind sicherlich damit verwandt, aber die Auswahl der Spiele ist komplexer. Es geht nicht nur um die Frage, welche Spiele gerade aktuell sind oder nachgefragt werden. Es geht auch um die Frage, welche Spiele in die individuelle digitale Strategie der jeweiligen Bibliothek passen. Standing Orders, auch wenn sie in Zusammenarbeit mit Bibliotheksmitarbeitern bearbeitet werden, die eine starke Gaming-Affinität haben, können die individuelle Situation vor Ort nicht überblicken. Der Bestandsaufbau im Bereich Gaming kann nicht der zentrale Teil einer Gaming-Strategie sein. Er ist vielmehr nur ein Teilelement einer Strategie, die auch Veranstaltungen, Informationsangebote etc. umfassen sollte. Zum anderen gibt die Bibliothek damit eine sehr große Chance aus den Händen, denn ein individueller Gaming-Bestand kann ein Alleinstellungsmerkmal sein.

Kommen wir nun zur Frage, wie denn nun eine sinnvolle Bestandsarbeit im Bereich Gaming konkret aussehen könnte. Zuerst sollten Sie sich überlegen, für welche Zielgruppe Ihre Gaming-Aktivitäten gedacht sind. Wie bereits angesprochen würde ich das Thema nicht auf männliche Jugendliche bzw. junge Erwachsene reduzieren. Ich hoffe, ich habe Ihnen im ersten Kapitel aufzeigen können, dass das Thema für eine weitaus größere Zielgruppe interessant sein könnte. Wenn wir nun wissen, welche Zielgruppe wir bedienen wollen und welche Spiele wir diesen Zielgruppen näher bringen möchten und warum, dann können wir uns überlegen bzw. recherchieren, welche Spiele hier überhaupt von einer Bibliothek verleihbar bzw. für den Bestand nutzbar sein könnten. Haben wir dies getan, müssen wir uns zum einen überlegen, wie wir mit den Spielen umgehen wollen, die wir in den Bestand aufnehmen möchten, und zum anderen müsste geklärt werden, welche Services im Umfeld der nicht im Bestand befindlichen Spiele für Gamer interessant sein könnten.

Aber gehören nur Games in den Gaming-Bestand? Nein! Gaming ist ein Meta-Thema. Es handelt nicht nur von Computerspielen. Die Gaming-Kultur und ihre Inhalte sind viel facettenreicher als es sich viele Menschen vorstellen können. Wenn wir also 
von einem Gaming-Bestand reden, dann müssen wir letztlich alle mit Gaming verbundenen Inhalte und Medien in den Bestand integrieren. Dazu gehören neben den eigentlichen Games u.a.:

- Sachbücher zum Thema Gaming: Hierbei handelt es sich um Bücher die einen Einstieg in die Welt des Gamings erlauben, und um Bücher, die bestimmte Aspekte des Gamings z. B. den Bereich Game-Based-Learning beleuchten. Diese Bücher sollten Sie unbedingt im Bestand haben. Eine Auswahl meiner Empfehlungen finden Sie auf meinem Blog. Diese Bücher sollten Sie aber auch selber lesen. Denn Sie helfen, das Thema Gaming zu verstehen und falls nötig wichtige Argumentationshilfen parat zu haben.

- Romane etc. aus dem Bereich Gaming: Es gibt eine Vielzahl an Romanen und vergleichbaren Werken, die direkt mit Computerspielen verbunden sind. Dies sind zum einen Bücher, die die Geschichten aus den Spielen weitererzählen bzw. im Universum des Spiels spielen. Zum anderen geht es um Bücher, die Vorlage für Computerspiele waren.

- Filme und Musik die mit Games verbunden sind. Wie auch bei Büchern, gibt es auch Filme und Musik, die direkt oder indirekt mit bestimmten Games verbunden sind.

- Gaming-Magazine: Es gibt eine Vielzahl an spannenden Gaming-Magazinen und Sie sollten versuchen, möglichst viele verschiedene in Ihrem Bestand zu haben. Dabei geht es nicht nur um die Mainstream-Magazine, sondern auch und vor allem um Magazine die ausgesuchte Nischenthemen behandeln. Ein gutes Beispiel ist sicherlich WASD. Hierbei handelt es sich um ein Magazin, in dem Spieler über Games bzw. ihr Verhältnis dazu schreiben.

- Lösungsbücher und Tutorials: Hierbei handelt es sich um z. T. um sehr detaillierte Abhandlungen zu einigen zumeist sehr komplexen Spielen.

- eBooks: Natürlich gibt es auch eBooks, die eine Verbindung mit Spielen haben. Hierbei gibt es zwei Sorten. Eine Variante sind die schon aufgezählten Bücher bzw. Bücher die in diese genannten Definitionen passen, aber nur als eBooks veröffentlicht werden. Die andere Variante ist etwas wirklich Spannendes: Es gibt eBooks, die Sammlungen von Texten aus Computerspielen sind. Ein sehr gutes Beispiel ist „The Imperial Library“. Hierbei handelt es sich um eine virtuelle Bibliothek mit Geschichten und Darstellungen aus dem Spiel „Skyrim - The Elder Scrolls“

Wie Sie sehen können, geht es bei der Bestandsarbeit zum Thema Gaming nicht nur um die Games an sich, sondern um eine Vielzahl an weiteren Inhalten.

Es ist nun wichtig zu überlegen, warum und für wen Sie Gaming anbieten möchten. Die Zielgruppeneinteilung in Altersklassen ist sicherlich ein erster wichtiger Schritt. Aber es ist ebenso wichtig zu überlegen, warum für die jeweilige Zielgruppe das Thema Gaming interessant sein könnte. Ein Beispiel: Wenn Sie definieren, dass Sie das Thema u. a. für die Zielgruppe der Erwachsenen zwischen 25 und 40 Jahren angehen wollen, dann müssen Sie überlegen, was diese Gruppe mit dem Thema machen soll. Geht es um Eltern, die sich mit dem Thema befassen, weil ihre Kinder in einem Alter sind, in dem sie Computerspiele spielen? Geht es um Erwachsene, die mit Games aufgewachsen sind und denen sie nun ein Stück ihrer Jugend zurückgeben möchten? Und wenn ja, sollen diese Erwachsenen vor allem neue Spiele kennenlernen oder sich mit ,ihren“ alten Spielen befassen? Möchten Sie vielleicht das Thema Gaming aus Sicht von Game-Based-Learning oder Gamification in Verbindung mit dem Beruf angehen und geht es dann noch um den Bestand? Wie Sie sehen können, gibt es eine Vielzahl von Zielgruppendefinitionen, die aber letztlich eine individuelle Strategie erfordern. 
In den letzten Jahren habe ich mir einige Bestände im Bereich Gaming in unterschiedlichen Bibliotheken angesehen. In den meisten Fällen konnte ich ein klares Muster erkennen. Man versuchte mit den vorhandenen Ressourcen ein Portfolio an Spielen zusammenzustellen, das möglichst Kinder und Jugendliche ansprach und zugleich auf den Plattformen gespielt wurde welche die genannte Zielgruppe vermeintlich am meisten nutzt. So findet man in vielen Bibliotheken Spiele für die Wii oder den Nintendo DS. Nicht so oft sind Spiele für die XBOX oder die Playstatíon $3 \mathrm{zu}$ finden. An sich ist diese Herangehensweise nicht falsch, jedoch sollte man sich immer überlegen, ob dieses Portfolio ausreicht. In vielen Bibliotheken wird zudem versucht, Spiele in den Bestand aufzunehmen, die gerade aktuell sind. Auch diese Idee ist nachvollziehbar, aber auf der anderen Seite nicht unbedingt zielführend. Wenn Sie versuchen, einen möglichst aktuellen Bestand zu haben, müssen Sie dies immer wieder leisten. Zudem können Sie die Frage, ob ein Spiel erfolgreich ist, erst nach ein paar Wochen nach der Veröffentlichung beantworten.

Ein gutes Negativ-Beispiel sind hier Spiele zu Filmen. Sehr oft glaubt man, dass Spiele zu Filmen und TV-Serien mehr oder weniger automatisch erfolgreich sein müssen, zumindest dann, wenn auch der Film erfolgreich ist. Aber dem ist nicht so. In den letzten Jahren konnte man immer wieder beobachten, dass Spiele zu Kinofilmen hinsichtlich Qualität und Gameplay schwächer waren als andere Titel. Natürlich gibt es auch Beispiele von erfolgreichen Spielen, welche auf Filmen oder TV-Serien basieren. Dies ist aber eher selten der Fall. Verstehen Sie mich bitte nicht falsch. Natürlich ist es wichtig, auch die aktuellen Spiele im Bestand zu haben, aber Sie sollten dafür den Markt intensiv beobachten. Nicht jedes Spiel, das auf den Markt kommt, ist auch nachhaltig erfolgreich. Da Bibliotheken aber in der Regel nur über begrenzte finanzielle Ressourcen verfügen, ist eine individuelle Auswahl wichtig.

Aber selbst wenn Sie einen interessanten Bestand haben - was macht ihn besonders? Was können Sie mit Ihrem Bestand anbieten, was vielleicht Gaming-Händler nicht anbieten können oder wollen? Wenn man sich die aktuelle Situation im stationären Einzelhandel ansieht, so kann man schnell zu dem Schluss kommen, dass man gegen die Elektronikfachmärkte und Gaming-Ketten keine Chance hat. Diese Anbieter verfügen über eine große Maße an aktuellen Spielen und in manchen Fällen bieten sie sogar den An- und Verkauf von gebrauchten Spielen an. Nun mögen einige Bibliotheksmitarbeiter einwenden, dass eine Bibliothek die Spiele ja mehr oder weniger kostenlos anbietet. In manchen Fällen fällt nur die Jahresgebühr an, in anderen Fällen kostet das Ausleihen von Games 1,- $€$ bis 2,- $€$. Egal welches Konzept Sie hier verfolgen. Ich behaupte auf der preislichen Ebene können Sie trotzdem kaum punkten. Natürlich hat eine Bibliothek auch eine soziale Funktion und natürlich ist es gut, wenn Sie den Menschen, die nicht über ausreichend finanzielle Mittel verfügen den Zugang zur Gaming-Welt ermöglichen. Trotzdem sollten Sie überlegen, was Ihren Bestand besonders macht. Vergessen Sie auch nicht, dass Sie einige Spiele aufgrund von Altersfreigaben oder bestimmten Inhalten gar nicht anbieten können oder wollen.

Ich empfehle Ihnen für den Bestandsaufbau bzw. die Bestandspflege folgendes Konzept:

1. Natürlich sollte es in Ihrem Bestand aktuelle Spiele geben. Achten Sie darauf, dass diese Spiele eine gute Chance auf dem Markt haben, d.h. Spiele, die bereits eine Historie aufweisen wie z. B. Pokemon oder Super Mario. Bei einigen Spielen ist dies nicht der Fall. Dann lohnt sich die Lektüre von Fachmagazinen und/oder das Gespräch mit einem Einkäufer eines Gaming-Händlers. Zudem sollten Sie die erfolgreichen Standard-Titel mehrfach im Angebot haben. So ist es sinnvoll, ei-

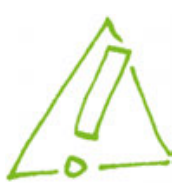


nen Titel wie Fifa 13 mehrfach im Angebot zu haben. Achten Sie aber unbedingt darauf, ob die Spiele überhaupt offline spielbar sind. Ansonsten macht das Verleihen keinen Sinn.

2. Dauerbrenner: Bestimmte Spiele sind auch dann noch interessant, wenn sie nicht aktuell sind. Dies können Spiele sein, die z. B. in Familien oder auf Partys beliebt sind, wie z.B. Singstar oder Guitar Hero oder Mario Kart. Es gibt auch Spiele, die über einen längeren Zeitraum erfolgreich sind, von denen Sie aber erst jetzt erfahren. So gab es mehrere Spiele zur TV-Cartoon-Serie „Kim Possible“ Der Erfolg vor allem der Nintendo DS-Version kam in kleinen Schritten. Irgendwann war die aktuelle Version schon kurz nach Erscheinen sehr erfolgreich. Mit dem Erfolg der aktuellen Version stiegen aber auch die Verkäufe der Vorgänger-Versionen. In so einem Fall ist es für eine Bibliothek sinnvoll, eben genau diese Versionen ebenfalls im Bestand zu haben. Das bedeutet aber, dass Sie auch das Umfeld des jeweiligen Spiels beleuchten müssen.

3. Retro-Games und Klassiker: Bei der Arbeit mit dem Thema Gaming wird sehr oft primär auf die aktuellen Titel geschaut. Dabei ist der Bereich Retro-Gaming (ich würde ihn um den Begriff „Klassiker“ erweitern) auf jeden Fall ein spannendes Thema. Wie gesagt ist das Thema Gaming nicht für Kinder und Jugendliche interessant. Es gibt viele Erwachsene, die, wenn auch nur durch ihre Kinder wieder mit dem Thema Gaming konfrontiert werden. Nur spielten diese Erwachsenen früher andere Spiele. Warum also versuchen wir nicht, dieser Zielgruppe die eigenen Spiele bzw. ihre eigene Gaming-Geschichte näher zu bringen?

\section{Hilfe gesucht}

Nun werden Sie zurecht einwenden, dass dies eigentlich gar nicht leistbar ist. Wie soll eine einzige Bibliothek all diese Informationen finden und verarbeiten? Bei sehr großen Bibliotheken mag dies noch möglich sein, aber kleine Bibliotheken haben schon mit dem Lektorat von Büchern genügend zu tun. Hierfür gibt es zwei Lösungen, die auch miteinander kombiniert werden können.

1. Ein Bibliotheks-Gaming-Bestands-Netzwerk

2. Ein Gaming-Bestands-Beirat

Beginnen wir mit der ersten Idee. Natürlich können Sie nicht alle relevanten GamingMagazine auf einmal beobachten. Und wenn es um den Bereich der Retro-Games geht, wird es noch schwieriger. Aber Sie können ein Netzwerk aufbauen, das Ihnen bei dieser Aufgabe hilft. So gibt es in Ihrer Umgebung mit Sicherheit die eine oder andere Gaming-Gruppe. Am besten Sie fragen im örtlichen Jugendclub und natürlich in der Schule bzw. bei den Eltern nach, die zu Ihnen kommen. Es gibt auch Communities zu einzelnen Spielen oder aber zu bestimmten Genres. Sie müssen einfach ein bisschen im Internet recherchieren. Ein Netzwerk aufzubauen, ist nicht einfach. Sie müssen zwei wesentliche Schritte gehen. Zum einen müssen Sie kontinuierlich dieses Netzwerk pflegen oder erweitern und zum anderen müssen Sie „zugeben“, dass Sie als Bibliothek nicht über ausreichende Kompetenz verfügen, um diese Aufgabe alleine zu meistern. Natürlich müssen Sie dann die Gamer, Eltern oder Gruppen persönlich ansprechen. Manchmal reicht es auch, wenn Sie online in einer oder mehreren Communities akzeptiert sind. Sie finden z.B. Gleichgesinnte in der FacebookGruppe „games4culture“. Sie können ebenso zusammen mit anderen Bibliotheken ein Gaming-Bibliotheks-Netzwerk aufbauen. Diese Herangehensweise eignet sich 
besonders für kleine und sehr kleine Bibliotheken. Wichtig ist nur, dass man vorab gemeinsam die Parameter für die Auswahl der Spiele festlegt.

Der zweite Ansatz geht letztlich in eine ähnliche Richtung. Auch hier bauen Sie ein Netzwerk auf, das Ihnen hilft, das Thema Bestandsarbeit nachhaltig zu bearbeiten. Allerdings bekommt dieses Netzwerk einen eigenen Namen und eine eigene „offizielle“ Aufgabe. Sie gründen einen Gaming-Bestands-Beirat, der einmal im Monat tagt und dabei gemeinsam überlegt, wie man den Bestand mit welchen Spielen erweitern kann. In dem Beirat sollten Gamer aller Altersklassen sitzen und natürlich Mitarbeiter Ihrer Bibliothek. Ein Pädagoge muss nicht unbedingt dabei sein, denn es geht hierbei nicht um pädagogisch wertvolle Spiele. Natürlich werden einigeRahmenbedienungen vorgegeben. Dies bezieht sich vor allem auf die Altersfreigaben, was z. B. bedeuten kann, dass Sie keine Spiele in den Bestand aufnehmen, die eine Altersfreigabe USK 16 oder USK 18 haben. Der Beirat sollte einmal im Monat offiziell tagen. Achten Sie darauf, dass nur solche Menschen Mitglied im Beirat werden können, die dem Thema Gaming gegenüber positiv eingestellt sind. Natürlich hat der Beirat auch Vorsitzende. Ich empfehle, einen Erwachsenen und ein Kind bzw. einen Jugendlichen als Vorsitzenden zu bestimmen. Der Vorsitz sollte alle sechs Monate wechseln. Die Vorsitzenden haben kein gesondertes Stimmrecht. Sie leiten nur die Treffen. Bei jeder Sitzung wird zuerst die aktuelle Situation im Bereich Gaming besprochen. Dazu gehören auch eventuelle Ausleihzahlen. Danach wird über die Vorschläge für Neuerwerbungen gesprochen. Vorschläge für Neuerwerbungen kann natürlich jeder machen. Dafür können Sie z. B. ein schwarzes Brett in der Bibliothek installieren. Die Bibliotheksmitarbeiter filtern dann die Spiele aus, die nicht den vorgegebenen Rahmenbedingungen entsprechen. Der Beirat sucht dann die Spiele aus, die angeschafft werden sollen. Nun kann es natürlich sein, dass weitaus mehr Spiele laut Beirat angeschafft werden sollen, als der Bibliothek finanzielle Ressourcen zur Verfügung stehen. Zudem ist es möglich, dass Sie gerade keine Zeit für Erwerbungen haben. Deshalb veröffentlichen Sie die Entscheidungen des Beirats und bitten die Bibliotheksnutzer, Ihnen bei der Anschaffung zu helfen. Dies kann auch mit gebrauchten Games geschehen.

Wie bereits erwähnt, sollten Sie nicht nur aktuelle Spiele in den Bestand aufnehmen. Deshalb möchte ich noch ein wenig detaillierter auf die Zielrichtung eingehen. Wenn es z.B. um Retro-Games geht, dann stellt sich die Frage, was Ihr Bestand eigentlich sein soll? Ist es ein klassischer Bibliotheksbestand? Kann es vielleicht mehr sein? Meine Empfehlung wäre, dass Sie sich als Bibliothek als Gaming- oder Spielort positionieren. Das bedeutet, dass Sie sowohl für Kinder und Jugendliche als auch für Erwachsene ein Angebot aufbauen. Am besten wäre es, wenn Sie ein kleines GamingArchiv aufbauen. Dabei geht es nicht darum, Games für die Nachwelt zu archivieren. Sie haben also nicht die Aufgabe, die Spiele zu pflegen bzw. zu sichern. Aber Sie können Ihren Bestand massiv erweitern, auch wenn es sich dabei um gebrauchte Spiele handelt. Wie gesagt: früher oder später werden Sie als Bibliothek in eine Situation kommen, in der es kaum noch möglich ist, Computerspiele in den Bestand aufzunehmen. Der digitale Vertrieb und die Bindung eines Spiels an einen Spieler macht dies nahezu unmöglich. Sie können aber mit den verbleibenden Spielen und zusammen mit weiteren Gaming-Aktivitäten ein interessantes Gaming-Portfolio aufbauen. 